\title{
EVIDÊNCIAS DO QUESTIONÁRIO “ADOÇÃO DO ÓLEO VEGETAL IN NATURA DE ACRÉSCIMO NA DIETA INFANTIL”: UMA REVISITA
}

\author{
Haydee Serrão Lanzillotti \\ Maria Elisa Barros
}

\section{RESUMO}

O objetivo do estudo foi reavaliar a estrutura do questionário "Adoção do óleo vegetal in natura de acréscimo na dieta infantil". Amostra: responsável pela alimentação das crianças $(n=300)$. Modelos: Análise Fatorial Confirmatória Estrita (AFCe), Análise Fatorial Exploratória (AFE), Análise Fatorial Exploratória/Confirmatória (E/AFC), Análise Fatorial Confirmatória (AFC). Validade convergente: Variância Média Extraída (VME) e validade discriminante (raiz VME). Confiabilidade: composite reliability (CR). Adesão à prática dietética: média dos escores brutos. Os resultados mostraram: Modelo SCFA: ajuste inadequado pelo Root Mean Square Error of Approximation (RMSEA=0,08); Modelo EFA: bidimensionalidade do questionário confirmado; Modelo E/CFA: limite superior de $\mathrm{RMSEA}=0,07$, modelo com reinspeção recomendada; Modelo CFA: RMSEA=0,02 (IC 90\%: 0,00-0,05, Prob=0,05), confirmado como modelo final, mostrando validades alcançadas: convergente (AVE: $F 1=0,7107$ e F2=0,8850) e discriminante (raiz VME: $F 1=0,8430$ e $F 2=0,9407$, correlação $F 1$ : $F 2=-0,1467$ ); e os coeficientes de confiabilidade foram: plausíveis $F 1 \quad(a=0,59)$ e satisfatórios: F2 $(a=0,80)$. Perfil de adesão: Itens positivos aprox. 2,5 , itens negativos aprox. 3.5 Redução de itens: 11 para 8 . Conclui-se que o modelo final, com duas dimensões, mostrou validade e confiabilidade.

\section{INTRODUÇÃO}

A adoção de práticas dietéticas recomendadas por orientação nutricional é um comportamento de difícil mensuração. Habilitar o maior número possível de profissionais de saúde a fazer uma primeira avaliação da aquiescência de recomendações do Ministério da Saúde, deixando as informações de avaliação nutricional para situações mais complexas, é uma possibilidade atraente. Neste sentido, aplicar instrumentos rápidos, mas válidos, pode auxiliar a nortear estratégias de intervenção nutricional. $O$ ato de medir é um componente essencial da pesquisa científica, seja nas ciências naturais, sociais ou da saúde. Segundo Streiner e Norman $^{1}$, existe atualmente maior conscientização sobre o impacto da saúde e dos cuidados de saúde na qualidade da vida humana e, para tanto, devem ser criados métodos para medir o que antes se pensava ser incomensurável. 
Desde 2013, o Ministério da Saúde adota como estratégia para combater o problema da desnutrição infantil o acréscimo de óleo vegetal in natura para o aumento da densidade energética da dieta. Esta recomendação se respalda na publicação dos "Dez passos para uma alimentação saudável", que está dirigida às mães e aos profissionais da saúde ${ }^{2}$. A proposta do Ministério se resume em adicionar o óleo vegetal cru em fórmulas lácteas ou em refeições infantis como substituo de formulações com densidade energética provenientes de amido e açúcares.

O acréscimo do óleo vegetal à dieta da criança tem o intuito de supri-la de ácidos graxos poliinsaturados essências, não sintetizados no organismo ${ }^{3}$. A introdução da alimentação complementar a partir dos seis meses ou até antes, sob a forma de papas de frutas e papas salgadas de hortaliças, reduz a ingestão destes nutrientes. No entanto, o óleo vegetal não deve ser aquecido à alta temperatura para evitar hidrólise, oxidação e polimerização ${ }^{4}$.

Lanzillotti et al. ${ }^{5}$ relatam que no atendimento nutricional ambulatorial presencia-se perplexidade entre os responsáveis pela alimentação da criança quando se prescreve o óleo de acréscimo por não fazer parte da rotina alimentar. Neste sentido, pode ser oportuno a criação de um instrumento de mensuração que seja capaz de identificar de forma mais fidedigna algumas das razões que justifiquem a resistência em não se adotar tal estratégia dietética e, por outro lado, possibilitar implementar o seu uso na prática nutricional pediátrica.

O objetivo deste estudo é reavaliar a estrutura dimensional do questionário "Adoção do óleo vegetal in natura de acréscimo na dieta infantil"

\section{MÉTODOS}

\section{Aspectos legais}

Este estudo faz parte do projeto de pesquisa "Óleo Vegetal Comestível na Dieta como Estratégia de Aumento da Densidade Energética" aprovado pelo Comitê de Ética em Pesquisa da Universidade do Estado do Rio de Janeiro (RJ), sob o protocolo número o melhor que consistente coloque. 006.3.2008. Após informações sobre os objetivos do estudo, os participantes assinaram o "Termo de Consentimento 
Livre e Esclarecido" sabendo que a qualquer momento poderiam declinar sua participação na pesquisa.

\section{Amostra e procedimentos}

Os participantes, responsáveis pela alimentação de crianças menores de dois anos que aguardavam consulta em sala de espera de atendimento ambulatorial em dois centros de atendimento pediátrico do Rio de Janeiro, Brasil, foram selecionados aleatoriamente. A coleta de dados ocorreu no período março de 2017 a janeiro de 2019. Foram considerados elegíveis responsáveis por preparar e/ou servir a refeição das crianças. Entre os responsáveis abordados, concordaram em participar da pesquisa 300 , sendo 150 de cada local.

Descrição do questionário "Adoção do óleo vegetal in natura de acréscimo na dieta infantil": o questionário consta de onze assertivas que expressam opiniões sobre a adição de óleo vegetal in natura na refeição de crianças para representar o construto "Adoção do óleo vegetal in natura de acréscimo na dieta infantil", delineado por duas dimensões: Dimensão1- "Benefícios do acréscimo de óleos vegetais in natura na dieta infantil" e Dimensão 2 "Possíveis problemas relacionados ao acréscimo de óleos vegetais in natura na dieta infantil" (Quadro1). O Questionário utilizou escala Likert, no qual as assertivas (itens) receberam escores em ordem decrescente, ou seja, 5 (concordo muito); 4 (concordo); 3 (nem concordo/nem discordo); 2 (discordo) e 1 (discordo muito). Cada item foi lido e, ao entrevistado, foi permitido escolher apenas um dos cinco escores da escala. Os itens foram nomeados segundo estudo exploratório realizado por Lanzillotti et $\mathrm{al}^{5}$.

Quadro1- Itens e dimensões do questionário para avaliar a adoção do óleo vegetal in natura de acréscimo na dieta infantil.

\begin{tabular}{|l|c|l|c|}
\hline Itens & Dimensão 1: & Itens & Dimensão 2: \\
& $\begin{array}{c}\text { Benefícios do acréscimo } \\
\text { de óleos vegetais in } \\
\text { natura na dieta infantil }\end{array}$ & & $\begin{array}{c}\text { Possíveis problemas } \\
\text { relacionados ao acréscimo } \\
\end{array}$ \\
& $\begin{array}{c}\text { de óleos vegetais in natura } \\
\text { na dieta infantil }\end{array}$ \\
\hline i4-P & $\begin{array}{c}\text { Colocar óleo vegetal cru } \\
\text { na comida da criança }\end{array}$ & i1-N & $\begin{array}{c}\text { Colocar óleo vegetal cru na } \\
\text { comida da criança piora a }\end{array}$ \\
\hline
\end{tabular}




\begin{tabular}{|l|c|c|c|}
\hline & $\begin{array}{c}\text { melhora a situação da } \\
\text { criança com baixo peso. }\end{array}$ & situação da criança com \\
baixo peso.
\end{tabular}

Nota: i: item; P: proposição de sentido positivo; N: proposição de sentido negativo Fonte: elaborado pelos autores.

\section{Análise de dados}

O estudo trata de um procedimento de análise confirmatória, uma vez que a modelação teórica sobre o construto já foi realizada previamente por Lanzillotti et $\mathrm{al}^{5}$.

Testou-se a normalidade multivariada entre as distribuições dos escores utilizando o teste de Doornik-Hansen ${ }^{6}$, um teste que usa assimetria e curtose. 
O exame da estrutura dimensional começou reavaliando a bidimensionalidade do questionário "Adoção do óleo vegetal in natura de acréscimo na dieta infantil" proposta por Lanzillotti et al ${ }^{5}$. Aplicou-se Análise Fatorial Confirmatória Estrita (AFCe) que utilizou equações estruturais ${ }^{7,8}$ com estimador maximum likelihood (ML) para obter soluções para os parâmetros do modelo recursivo, ou seja, unidirecionalidade das relações de determinação entre as variáveis ${ }^{9}$.

Modelos de Equações Estruturais (MEE) permitem o teste confirmatório da estrutura psicométrica de escalas de medida permitindo analisar relações explicativas entre múltiplas variáveis simultaneamente, sejam essas latentes ou observadas ${ }^{10}$. Iniciou-se com o modelo de mensuração multifator AFCe considerando a bidimensionalidade da estrutura fatorial do questionário. A adequação do ajustamento - Goodness of fit (GOF) foi avaliada usando três índices: a) Root Mean Square Error of Approximation (RMSEA), o qual incorpora uma função de penalidade para parcimônia pobre do modelo $8,11,12$ e cujos valores abaixo de 0,06 sugerem um ajuste (adequado), enquanto valores acima de 0,10 indicam ajuste inadequado e rejeição do modelo ${ }^{11}$ ou reespecificação; ; ) Comparative Fit Index (CFI) e c) Tucker-Lewis index (TLI), os quais representam índices de ajustamento incremental ${ }^{7,8}$ contrastando 0 modelo hipotetizado para um modelo baseline aninhado mais restrito, "null model'. Ambos variam de zero a um e valores maiores que 0,9 são indicativos de ajuste adequado $^{8,13}$.

A fim de auxiliar a reespecificação foi utilizado o Modification Index (MI). Um MI reflete quanto um modelo geral qui-quadrado diminui se um parâmetro restrito é estimado livremente. O Ml é aproximadamente equivalente à diferença no quiquadrado entre dois modelos, em que em um modelo o parâmetro é fixo ou restrito e no outro o parâmetro é estimado livremente. Relações entre erros de medição de itens envolvendo valores de $\mathrm{Ml}$ igual ou superior a 10 foram analisados mais detalhadamente, paralelamente com a grandeza do expected parameter changes (EPC) correspondente a alterações para parâmetros livremente estimados ${ }^{8}$.

Uma vez estabelecido os itens que seguiram para a reespecificação do modelo, iniciou-se por AFE para reavaliar dimensionalidade do questionário.

Antecipando um possível desajuste do modelo e prevendo estruturas dimensionais alternativas plausíveis, o próximo passo consistiu em reexplorar a estrutura dimensional do questionário por meio de um procedimento de modelo de 
equação estrutural exploratório, como proposto por March et $\mathrm{al}^{14}$. Isto consiste em ajustar uma sequência de modelos exploratórios, dentro de uma estrutura de AFC $(\mathrm{E} / \mathrm{AFC})^{8}$. Nas análises exploratórias foram inferidos como itens satisfatórios os que apresentaram cargas fatoriais $(\lambda)$ maior $0,40^{15}$. Foram considerados itens com cargas cruzadas aqueles com cargas fatoriais similares em dois ou mais fatores em um mesmo item e cuja diferença entre as cargas fosse menor do que $0,10^{16}$. A variância do erro (uniqueness) foi considerada adequada quando a magnitude se apresentou menor ou igual a $0,50^{15}$. A plausibilidade teórica dos itens também foi considerada para avaliar o padrão e o número de fatores. Os mesmos índices GOF (RMSEA, CFI e TLI) apresentados anteriormente foram utilizados para avaliar a qualidade do ajustamento. A reavaliação de cargas cruzadas e correlações residuais foram realizadas usando MI e EPC, sendo aplicados os mesmos critérios.

Uma vez definido o modelo, em prosseguimento foi avaliada a validade fatorial convergente e discriminante baseada em fatores ${ }^{7,8}$. Ambos são baseados na Average Variance Extracted (AVE) ${ }^{8,16,17 .}$

A AVE avalia a quantidade de variância capturada por um fator comum em relação à quantidade de variância devido a erro de medição aleatório ${ }^{15}$. É uma função da relação entre a carga/fator/item padronizada e o erro de mensuração relacionado (uniqueness) que se refere à parte de um indicador não explicado pelo fator latente ${ }^{18}$. Os valores variam de 0 a 1 . Um fator mostra validade convergente se AVE igual ou maior que 0,50 , o que é indicativo de que pelo menos $50 \%$ da variação em uma medida é devido à hipótese característica subjacente. Validade convergente baseada em fatores é questionável se AVE se apresentar menor que 0,50 , pois a variância devido ao erro de mensuração é então maior que a variância devida ao construto ${ }^{15}$. Em modelos multidimensionais, a validade discriminante baseada em fator é considerada segura se, para um dado fator, a raiz quadrada de sua AVE está acima das correlações com quaisquer outros fatores relacionados no modelo ${ }^{19}$.

A confiabilidade foi avaliada pela composite reliability (CR), dada por:

$$
C R=\frac{\left(\sum_{i=1}^{i=k} \chi i\right)}{\left(\sum_{i=1}^{i=k} \lambda i\right)+\left(\sum_{i=1}^{i=k} v \delta i\right)}
$$

Onde

$\lambda i$ são cargas fatoriais

ठi variância do erro para $k$ itens. 
A confiabilidade composta varia de 0 a 1 sendo considerada satisfatória, quando $\geq 0,70^{8}$.

A fim de desenhar o perfil de disposição dos entrevistados para adoção da prática recomendada pelo Ministério de Saúde, foram utilizados os escores brutos do modelo final e calculado média e intervalo de confiança. A hipótese de trabalho é descrita da seguinte forma: Se os participantes estivessem dispostos a adotar o uso do óleo vegetal in natura para o aumento da densidade energética da dieta, entre outros benefícios, esperava-se que os itens da Dimensão 1: "Benefícios do acréscimo de óleos vegetais in natura na dieta infantil" recebam escores médios altos, e da Dimensão 2: "Possíveis problemas relacionados ao acréscimo de óleos vegetais in natura na dieta infantil", escores médios baixos.

\section{RESULTADOS}

Participaram do estudo 300 responsáveis pela alimentação de crianças menores de dois anos que aguardavam a consulta nas salas de espera de ambulatórios de pediatria, sendo 150 de uma operadora de planos de saúde em São João de Meriti (SJM) e 150 da Policlínica Piquet Carneiro da Universidade do Estado do Rio de Janeiro (PPC/UERJ). Em SJM a idade média dos responsáveis foi 38,6 anos $(\mathrm{dp}=12,20 ; \min =18$ e $\max =66)$. Quanto ao gênero, $87,33 \%$ do sexo feminino e $12,67 \%$ do sexo masculino. Em PPC/UERJ, a idade média foi 46,84 anos ( $\mathrm{dp}=14,12$; $\min =20$ e $\max =78$ ). Quanto ao gênero, $84 \%$ do sexo feminino e $16 \%$ do sexo masculino. Em relação à escolaridade, foi predominante $3^{\circ}$ grau completo e $2^{\circ}$ grau completo para SJM e PPC/UERJ, respectivamente.

O teste de Doornik-Hansen confirmou a normalidade multivariada dos escores relativos aos itens do questionário (chi2 (22)=783.910; Prob>chi2 $=0.0000$ ).

A solução fatorial iniciou com AFCe do modelo A que apresentou valores de CFI e TFI de 0,997 e 0,995, respectivamente, os quais indicam ajuste adequado. $O$ RMSEA alcançou valor de 0,08 com limite superior de 0,10 (Prob=0,05), quando se espera estimativa de ponto abaixo de 0,06. Este achado infere sobre um ajuste inadequado (Tabela 1). 
Tabela 1 - Modelo A: Análise Fatorial Confirmatória estrita (AFCe), Modelo B1: Análise Fatorial Exploratória (AFE), Modelo B2: Análise Fatorial Exploratória/Confirmatória (E/AFC), Modelo D: Análise Fatorial Confirmatória (AFC) do questionário "Adoção do óleo vegetal in natura de acréscimo na dieta infantil", Rio de Janeiro 2017-2019.

\begin{tabular}{|c|c|c|c|c|c|}
\hline \multirow{3}{*}{ Item } & \multicolumn{5}{|c|}{ Modelo A: AFCe } \\
\hline & Fator 1 & & Item & Fator 2 & \\
\hline & $\lambda$ & $\varepsilon$ & & $\lambda$ & $\varepsilon$ \\
\hline i4P & $0,8122(0,7607-0,8636)$ & 0,3402 & $i 1 \mathrm{~N}$ & $0,5940(0,5159-0,6721)$ & 0,6471 \\
\hline i6P & $0,7723(0,7150-0,8296)$ & 0,4034 & $i 3 N$ & $0,8719(0,8371-0,9066)$ & 0,2397 \\
\hline i7P & $0,6613(0,5878-0,7348)$ & 0,5626 & $\mathrm{i} 8 \mathrm{~N}$ & $0,9245(0,8961-0,9528)$ & 0,1452 \\
\hline i9P & $0,4804(0,3842-0,5766)$ & 0,7691 & $\mathrm{i} 14 \mathrm{~N}$ & $0,8573(0,8196-0,8949)$ & 0,2650 \\
\hline ¡10P & $0,7389(0,6765-0,8013)$ & 0,4539 & & & \\
\hline i13P & $0,5698(0,4841-0,6554)$ & 0,6753 & & & \\
\hline i15P & $0,5968(0,5143-0,6793)$ & 0,6437 & & & \\
\hline F1-F2 & $-0,1211$ & & & & \\
\hline RMSEA & $0,08(0,07-0,10)$ & & & & \\
\hline $\mathrm{CFI}$ & 0,93 & & & & \\
\hline \multirow[t]{2}{*}{ TLI } & 0,92 & & & & \\
\hline & \multicolumn{5}{|c|}{ Modelo B1: AFE } \\
\hline \multirow[t]{2}{*}{ Itens } & Fator 1 & & Item & Fator 2 & \\
\hline & $\lambda$ & $\varepsilon$ & & $\lambda$ & $\varepsilon$ \\
\hline i4P & 0,8470 & 0,3380 & $\mathrm{i} 1 \mathrm{~N}$ & 0,5222 & 0,6172 \\
\hline i6P & 0,7403 & 0,4399 & $\mathrm{i} 3 \mathrm{~N}$ & 0,8660 & 0,2564 \\
\hline i7P & 0,6811 & 0,5520 & i8N & 0,9514 & 0,1404 \\
\hline i9P & 0,5303 & 0,7533 & $\mathrm{i} 14 \mathrm{~N}$ & 0,8783 & 0,2506 \\
\hline ¡10P & 0,7375 & 0,4540 & & & \\
\hline ¡13P & 0,5497 & 0,6673 & & & \\
\hline i15P & 0,5474 & 0,5938 & & & \\
\hline $\mathrm{F} 1-\mathrm{F} 2$ & 0,4635 & & & & \\
\hline RMSEA & $\ldots$ & & & & \\
\hline $\mathrm{CFI}$ & $\ldots$ & & & & \\
\hline
\end{tabular}


TLI

\begin{tabular}{|c|c|c|c|c|c|}
\hline \multirow{3}{*}{ Item } & \multicolumn{5}{|c|}{ Modelo B2: E/AFC } \\
\hline & Fator1 & & Itens & Fator2 & \\
\hline & $\lambda$ & $\varepsilon$ & & $\lambda$ & $\varepsilon$ \\
\hline i4P & $0,8025(0,7494-0,8555)$ & 0,3559 & i3N & $0,8828(0,8487-0,9169)$ & 0,2205 \\
\hline i6P & $0,7898(0,7349-0,8448)$ & 0,3761 & $\mathrm{i} 8 \mathrm{~N}$ & $0,9154(0,8853-0,9454)$ & 0,1620 \\
\hline i7P & $0,6526(0,5776-0,7275)$ & 0,5740 & $\mathrm{i} 14 \mathrm{~N}$ & $0,8569(0,8194-0,8945)$ & 0,2655 \\
\hline i10P & $0,7359(0,6731-0,7987)$ & 0,4583 & & & \\
\hline i13P & $0,5751(0,4899-0,6602)$ & 0,6692 & & & \\
\hline i15P & $0,5952(0,5121-0,6783)$ & 0,6456 & & & \\
\hline F1-F2 & $-0,1561$ & & & & \\
\hline
\end{tabular}

$\begin{array}{cc}\text { RMSEA } & 0,05(0,02-0,07) \\ \text { CFI } & 0,98 \\ \text { TLI } & 0,97\end{array}$

\begin{tabular}{|c|c|c|c|c|c|}
\hline \multirow[b]{2}{*}{ Item } & \multicolumn{5}{|c|}{ Modelo D: AFC } \\
\hline & Fator1 & & Itens & Fator2 & \\
\hline & $\lambda$ & $\varepsilon$ & & $\lambda$ & $\varepsilon$ \\
\hline i4P & $0,8003(0,7455-0,8551)$ & 0,3594 & i3N & $0,8830(0,8488-09171)$ & 0,2202 \\
\hline i6P & $0,8135(0,7604-0,8666)$ & 0,3381 & i8N & $0,9152(0,8851-0,9454)$ & 0,1622 \\
\hline i7P & $0,6419(0,5644-0,7194)$ & 0,5879 & $i 14 N$ & $0,8569(0,8193-0,8946)$ & 0,2655 \\
\hline i10P & $0,7335(0,6694-0,7976)$ & 0,4618 & & & \\
\hline i13P & $0,5641(0,4770-0,6512)$ & 0,6817 & & & \\
\hline F1-F2 & $-0,1467$ & & & & \\
\hline RMSEA & $0,02(0,00-0,05)$ & & & & \\
\hline CFI & & & & & \\
\hline TLI & 0,99 & & & & \\
\hline
\end{tabular}

Modelo C: Modelo Exploratório tridimensional: Autovalores de 4,32; 1,67 e 0,37, para Fator 1, Fator 2 e Fator 3, respectivamente, o que não recomenda a tridimensionalidade do instrumento. F1: fator 1, F2: fator $2, \lambda$ : cargas fatoriais padronizadas, em parênteses: $95 \%$ intervalo de confiança, $\varepsilon$ : erros de mensuração (variância do erro), F1-F2: correlação de fatores, RMSEA: Root Mean Square Error of Approximation, em parênteses: $90 \%$ intervalo de confiança, CFI: Comparative Fit Index, TLI: Tucker-Lewis Index. 
O modelo A: AFCe necessitou de ajustes, como observado por RMSEA, embora apresentasse cargas fatoriais aceitáveis nos dois fatores, mas variâncias do erro altas nos itens i7P, i9P, i13P, i15P do fator 1 e i $1 \mathrm{~N}$ do fator 2 (Tabela 1). Os Ml indicam que o modelo podia ser mais bem especificado, tendo em vista MI de 12,20 na covariância entre i6P- i9P (Tabela 2), ou seja, que o erro da interação entre os referidos itens reduziria em pelo menos 12,20 o valor de qui-quadrado e a estimativa do novo parâmetro incorporado ao modelo (EPC) foi -0,2395. Uma vez que se trata de instrumento novo de mensuração, o modelo foi reespecificado para se reexplorar a estrutura dimensional do questionário.

Em prosseguimento à $\mathrm{AFCe}$, Modelo $A$, três estudos foram realizados por AFE, o Modelo B1: AFE, constrangido a dois fatores, com desdobramento para o Modelo B2: E/AFC e o Modelo C: AFEt (Análise Fatorial Exploratória tridimensional), este modelo numa tentativa da possibilidade de mais um fator.

No Modelo B1: AFE, observou-se no Fator 1 autovalor (eigenvalue) de 4,28 e no Fator 2, valor de 1,64, o que possibilitou seu desdobramento no Modelo B2: E/AFC. No entanto o Modelo C: AFEt, por apresentar autovalores de 4,32; 1,67 e 0,37, para Fator1, Fator 2 e Fator 3, respectivamente (dados não apresentados em tabela informados no rodapé da tabela 1). O autovalor do Fator 3 , menor que 1, não recomenda a tridimensionalidade do instrumento, não sendo mais explorado o Modelo C: AFEt.

A estrutura fatorial do Modelo B1: AFE abarca no, Fator 1, todos os itens de caráter positivo, Dimensão 1, no entanto os itens i7P, i9P, i13P e i15P apresentaram variâncias de erros expressivas, especialmente o item i9P (Tabela 1). Em relação aos itens de caráter negativo, Dimensão 2, todos os itens alcançaram cargas fatoriais adequadas, mas o item i1 $\mathrm{N}$ apresentou variância de erro relativamente alta $(\varepsilon=0,6172)$ e muito afastada das dos demais itens, cuja variância do erro variou entre 0,1404 a 0,2564 (Tabela 1). Dando prosseguimento ao estudo exploratório na perspectiva (E/AFC), foi criado o Modelo B2: E/AFC, sem os itens i9P e i1N, tendo em vista que o item i9P, com variância de erro alta $(0,7691)$ covariou com o item i6P apresentando MI de 12,20 (Tabela 2), acima do esperado ( $\mathrm{Ml}=10)$, conforme aludido anteriormente. Os itens i7P, i13P e i15P, apresentaram variância de erro limítrofes ao ponto de corte $(\varepsilon=0,50)$, ou seja, $0,5626,0,6753$ e 0,6437 , respectivamente (Tabela1). Em relação ao $\mathrm{Ml}$, os itens i7P e i13P não covariaram com outros itens, o que recomenda sua 
permanência para verificar seu comportamento no prosseguimento do estudo fatorial exploratório. O item i15P covariou com os itens i6P e i9P com Ml de 8,00 e 6,34, indicando também sua permanência no seguimento exploratório.

Tabela 2 - Modification Index (MI) e Expected Parameter Changes (EPC) da sequência de modelos do questionário "Adoção do óleo vegetal in natura de acréscimo na dieta infantil”, Rio de Janeiro 2017-2019

\begin{tabular}{|c|c|c|c|c|c|c|c|c|}
\hline \multicolumn{3}{|c|}{ Modelo A: AFCe } & \multicolumn{3}{|c|}{ Modelo B: E/AFC } & \multicolumn{3}{|c|}{ Modelo D: AFC } \\
\hline Covariância & $\mathrm{MI}$ & EPC & Covariância & $\mathrm{Ml}$ & EPC & Covariância & $\mathrm{MI}$ & EPC \\
\hline e. i4P - e. i6P & 7,24 & 0,2568 & e. i4P - e. i6P & 5,24 & 0,22 & e. i6P - e. i7P & 5,24 & $-0,22$ \\
\hline e. i4P - e. i1N & 4,82 & $-0,1495$ & e. i6P - e. i7P & 3,84 & $-0,15$ & & & \\
\hline e.i6P - e. i9P & 12,2 & $-0,2395$ & e.i6P - e.i15P & 11,76 & $-0,25$ & & & \\
\hline e.i6P - e. i10P & 4,57 & 0,1747 & e.i15P - e.i8N & 4,72 & $-0,16$ & & & \\
\hline e.i6P - e. i15P & 8,00 & $-0,2027$ & & & & & & \\
\hline e.i6P - e.i3N & 4,48 & $-0,1556$ & & & & & & \\
\hline e.i9P - e.i15P & 6,34 & 0,1564 & & & & & & \\
\hline e.i9P - e.i3N & 4,5 & 0,1431 & & & & & & \\
\hline e.i9P - e.i8N & 7,07 & $-0,2092$ & & & & & & \\
\hline e.i1N - e.i3N & 3,879 & $-0,1423$ & & & & & & \\
\hline e.i8N - e.i14N & 3,879 & $-0,4372$ & & & & & & \\
\hline
\end{tabular}

AFCe: Análise Fatorial Confirmatória Estrita, E/AFC: Análise Fatorial Exploratória/Confirmatória, AFC: Análise fatorial Confirmatória, i: item, P: positivo (Dimensão 1- "Benefícios do acréscimo de óleos vegetais in natura na dieta infantil"), N: negativo (Dimensão 2- "Possíveis problemas relacionados ao acréscimo de óleos vegetais in natura na dieta infantil”), MI: significância 0,05.

Neste modelo, B2: E/AFC, as cargas fatoriais foram adequadas para as duas dimensões, com variâncias de erro altas para os itens i7P, i13P e i15P. Os índices de ajuste com valores de 0,984 e 0,978 para CFI e TFI, respectivamente, indicaram ajustamento adequado. A estimativa de ponto para RMSEA foi de 0,05 variando de 0,02 a 0,07 (Prob:0,05). No entanto, o limite superior de RMSE com valor de 0,07 recomendou inspecionar mais atentamente o modelo. A covariância entre os itens i6P e i15P mostrou MI de 11,76 com parâmetro de restrição relaxada (EPC) igual a -0,25 (Tabela 2). Examinando as cargas fatoriais destes itens, verificou-se que i15P apresentou 0,5952 (IC 95\%: 0,5121-0,6783) e i6P 0,7898 (IC 95\%:0,7349-0,8448). As 
variâncias de erro foram 0,6456 e 0,3761, respectivamente (Tabela 1), indicando a retirada do item i15P. Os achados conduziram a retirada do item i15P e permanência do i6P. O modelo B2: E/AFC foi reespecificado, criando o Modelo D.

O Modelo D: AFC mostrou RMSEA de 0,02 (IC 90\%: 0,00-0,05, Prob=0,05). Uma comparação com o Modelo B: E/AFC (RMSEA 0,05: IC 90\% 0,02-0,07) mostrou melhoria de ajuste. Neste modelo as cargas fatoriais foram expressivas em ambos os fatores considerando o ponto de corte de 0,40 . No entanto, em relação à variância do erro, os itens i7P $(\varepsilon=0,5879)$ e i13P $(\varepsilon=0,6817)$ apresentaram valores acima do esperado $(\varepsilon=0,50)$. Mais uma vez o item i7P ficou numa situação limítrofe com carga fatorial adequada, mas variância do erro pouco acima do ponto de corte $(0,50)($ Tabela 1).

Analisando a semântica do item i13P ("O acréscimo de óleo vegetal cru não eleva o colesterol da criança") em relação ao intuito de aumento da densidade energética da dieta e à questão teórica em relação aos outros benefícios do acréscimo do óleo vegetal in natura, optou-se pela permanência do item i13P.

$\mathrm{A}$ análise de possíveis correlações residuais vistas por $\mathrm{Ml}$, identificou apenas a covariância i6P- i7P, apresentando $\mathrm{Ml}=5,24$, o que não traria vantagem acentuada para o ajustamento do modelo a retirada tanto de i7P quanto do item i13P (Tabela 2).

Os fatores, F1 (Dimensão 1) e F2 (Dimensão 2), apresentaram validade fatorial convergente com valores de AVE de 0,7107 e 0,8850, respectivamente. A validade fatorial discriminante também foi alcançada tendo em vista que a raiz quadrada da AVE dos respectivos fatores está acima da correlação entre o Fator $1 \mathrm{e}$ Fator 2 (Tabela 3).

Em relação à composite reliability, valores de 0,59 e 0,80, para os fatores $1 \mathrm{e}$ 2 respectivamente, permitem inferir como satisfatória a confiabilidade para Fator 2. No entanto, a confiabilidade para o Fator 1 pode ser considerada plausível (Tabela 3). 
Tabela 3 - Validade Average Variance Extracted (AVE), raiz quadrada da AVE (correlação entre fatores) e Confiabilidade (composite reliability) para os fatores 1 e 2 do questionário "Adoção do óleo vegetal in natura de acréscimo na dieta infantil", Rio de Janeiro 2017-2019

\begin{tabular}{lcc}
\hline & \multicolumn{2}{c}{ Validade } \\
Fator 1 & AVE & Variância \\
& $\sqrt{A V E}$ & 0,7107 \\
Fator 2 & $\mathrm{AVE}$ & 0,8430 \\
& $\sqrt{A V E}$ & 0,8850 \\
Correlação de fator & $\phi 1-2$ & 0,9407 \\
\hline & Confiabilidade & $-0,1467$ \\
Fator 1 & composite reliability & 0,59 \\
Fator 2 & & 0,80 \\
\hline
\end{tabular}

Valores baseados sobre dois fatores da solução do Modelo D: AFC na Tabela 1

A média dos itens de caráter positivo, Dimensão 1: "Benefícios do acréscimo de óleos vegetais in natura na dieta infantil" apresentaram valores menores que 2,5, à exceção do item i13P ("O acréscimo de óleo vegetal cru não eleva o colesterol da criança") com valor ligeiramente mais alto $(2,56)$, tendendo a não concordância. Em relação aos itens de caráter negativo, Dimensão 2: "Possíveis problemas relacionados ao acréscimo de óleos vegetais in natura na dieta infantil", os escores médios apresentaram valores ao redor de 3,5, ou seja, tendência à concordância (Tabela 4).

Tabela 4 - Escores médios e Intervalo de Confiança das respostas aos itens do Modelo D-AFC do questionário "Adoção do óleo vegetal in natura de acréscimo na dieta infantil", Rio de Janeiro 2017-2019

\begin{tabular}{cccccc}
\hline Item & Média & IC & Item & Média & IC \\
\hline i4P & 2,45 & $2,36-2,53$ & i3N & 3,56 & $3,47-3,64$ \\
i6P & 2,45 & $2,36-2,53$ & i8N & 3,56 & $3,47-3,64$ \\
i7P & 2,49 & $2,40-2,57$ & i14N & 3,57 & $3,49-3,66$ \\
i10P & 2,43 & $2,35-2,51$ & & & \\
i13P & 2,56 & $2,46-2,65$ & & &
\end{tabular}

AFC: Análise Fatorial Confirmatória, IC: Intervalo de Confiança (nível de significância $=0,05$ ) 
Os resultados permitiram construir o questionário final com oito itens distribuídos em duas dimensões, a saber: Dimensão 1: i4P (Colocar óleo vegetal cru na comida da criança melhora a situação da criança com baixo peso), i6P (Usar óleo vegetal cru na comida da criança melhora a imunidade da criança), i7P (Se eu acrescentar óleo vegetal cru na comida da criança, ela não ficará obesa), i10P (Usar óleo vegetal cru na comida da criança melhora sua resistência a doenças transmissíveis) i13P (O acréscimo de óleo vegetal cru não eleva o colesterol da criança) e Dimensão 2: i3N (Colocar óleo vegetal cru na comida da criança causa náuseas), i8N (Colocar óleo vegetal cru na comida da criança causa vômito) i14N (O uso de óleo vegetal cru todos os dias provoca diarreia).

\section{DISCUSSÃO}

Conforme apresentado na introdução, este estudo se propôs reavaliar a estrutura dimensional do questionário "Adoção do óleo vegetal in natura de acréscimo na dieta infantil”. Esta prática dietética é uma recomendação do Ministério da Saúde ${ }^{2}$ para aumentar a densidade energética da dieta. Trata-se de um instrumento que pode alcançar um grande número de seguidores ao longo dos anos, bem como surgir algum desacordo sobre sua dimensionalidade, quando de sua replicação em outros cenários. Por extensão, poderão surgir questões sobre a distribuição interna de itens. Estas observações levam a sua necessária replicação para avançar e examinar algumas propriedades que possam não ter sido avaliadas anteriormente.

No que se refere à amostra, o questionário analisado neste estudo possui 11 variáveis e foi aplicado em 300 indivíduos, obtendo-se a relação variável/sujeito igual a 27. Segundo Hair et $\mathrm{al}^{15}$, para uma significância de 0,05 , tamanhos de amostras entre 250 a 350 e a carga fatorial entre 0,30 a 0,35 devem ser exigidas. Neste estudo foram usadas cargas fatoriais $(\lambda)$ maiores que 0,40 . Amostras robustas tendem a fornecer resultados mais precisos, diminuindo o efeito do erro amostral ${ }^{17} \mathrm{e}$ amostras modestas $(\leq 100)$ podem levar a efeitos fracos nos dados, comprometendo índices de ajuste (GOF), MI e EPC 8 . GOF são frequentemente afetados por vários aspectos da situação analítica, como tamanho da amostra, complexidade do modelo, método de estimativa (estimador maximum likelihood), normalidade dos dados e tipo de dados. Neste estudo foram utilizadas variáveis em escala ordinal, necessitando avaliar a 
normalidade multivariada das variáveis. O teste de Doornik-Hansen (2008) corroborou tal evidência mostrando o qui-quadrado com valor adequado (chi2 (22)=783.91, Prob $>$ chi2 $=0.0000$ ) .

A análise fatorial do modelo $A$ e seus desdobramentos até o Modelo D: AFC permitiu confirmar a estrutura dimensional relatada anteriormente na literatura por estudos exploratórios utilizando rotação obliqua $\operatorname{Promax}^{5}$ e rotação ortogonal Quartimax 20 .

No modelo A o resultado do RMSEA $(0,08)$ exigiu a busca por MI e EPD. O MI de 12,20 e EPC de -0,2395 na covariância entre i6P-i9P mostraram redução da estimativa do qui-quadrado e alteração dos parâmetros, que podem ser considerados não parcimoniosos. No entanto, Brown ${ }^{8}$ alerta para que toda a reespecificação de um modelo deve ser justificada com base em pesquisas ou teorias e acrescenta que vários estudos destacaram problemas que ocorrem quando os modelos são reespecificados somente com base em MI e EPC. Para o autor, o cuidado com a plausibilidade teórica melhora muito o sucesso da pesquisa de especificação. Neste sentido, a semântica dos itens foi avaliada, buscando-se as assertivas: item i6P ("Usar óleo vegetal cru na comida da criança melhora a imunidade da criança") e i9P ("É bom para a criança colocar óleo vegetal cru na mamadeira"). Consubstanciado na fundamentação teórica e nas Políticas de Atenção à Saúde Materno-Infantil que incentivam o aleitamento materno, existiu plausibilidade teórica para a retirada do item i9P, uma vez que a assertiva pode ir de encontro com orientações das políticas de saúde materno infantil. Neste modelo, os itens i1N "Colocar óleo vegetal cru na comida da criança piora a situação da criança com baixo peso" e i4P "Colocar óleo vegetal cru na comida da criança melhora a situação da criança com baixo peso", são itens inversamente relacionados, ou seja, de sentido contrário. Segundo Streiner e Norman ${ }^{1}$ assertivas com sentido negativo tendem a reduzir a validade do instrumento. Os achados de análise de dados e considerações teóricas exigiram a resepecificação do modelo, prática adotada em instrumento novo de mensuração ${ }^{8}$.

No desdobramento do Modelo $A$ os resultados da análise fatorial do Modelo B1: AFE mostraram que se justificava a retirada dos itens i9P e i1 N, tendo em vista suas variâncias de erro. Seguindo-se a análise, criou-se o Modelo B2: E/AFC, que apresentou RMSEA de estimativa de ponto de 0,05, mas com limite superior do intervalo de confiança alcançado 0,07 . Neste modelo, o item i15P foi o indicado a ser 
retirado do questionário, fundamentado também na covariância entre os itens i6P ("Usar óleo vegetal cru na comida da criança melhora a imunidade da criança") e i15P ("O certo é não refogar os alimentos da criança e colocar o óleo vegetal cru depois da papa salgada pronta") mostrou MI de 11,76, com o item i6P apresentando melhor desempenho. Não refogar os alimentos em culinária é uma atitude muito diferente da herança cultural de quem prepara alimentos. No Brasil, o termo refogado é comumente utilizado para mistura de cebola e alho fritos em pouco óleo e cozimento em fogo baixo ${ }^{21}$. Por outro lado, o acréscimo de óleo como substância alimentar que pode melhorar a imunidade da criança é prática recomendada por um profissional de saúde; é algo a ser pensado. Evocando estas possíveis argumentações, e as evidências das cargas fatoriais e variâncias do erro, o item i15P foi retirado.

Finalmente o modelo D: AFC, no qual o item i13P ("O acréscimo de óleo vegetal cru não eleva o colesterol da criança") foi o item no qual a variância do erro manteve-se alta $(\varepsilon=0,6817)$.

Streiner e Norman ${ }^{1}$ relatam que itens com palavras negativas tendem a reduzir a validade do instrumento. No presente estudo esta questão foi criada para avaliar a carga negativa relativa ao comportamento dos indivíduos na contemporaneidade em relação às gorduras. Azevedo (2015) aludindo a pressupostos da Antropologia e Sociologia da Alimentação e da Nutrição, faz o leitor refletir sobre a demonização dos alimentos. Diz a autora que na década de 1960 aconteceu a mais peculiar demonização de um alimento - o leite materno. "Pediatras passaram a recomendar a substituição do leite "fraco" das mamíferas humanas pelo leite desidratado e maternizado" (p.721) 22. Em relação à gordura, o lema é "gordura animal que satura o sangue" (p.720) ${ }^{22}$. A questão da demonização dos alimentos, é que no caso das gorduras, o conhecimento do senso comum não distingue de forma apropriada a diferença entre gorduras saturadas, monossaturadas e poli-insaturadas. Mesmo que na elaboração do item tenha sido utilizada a expressão "não eleva o colesterol", "não" palavra negativa, por razões contextuais, o item permaneceu no questionário sem alteração semântica.

A validade fatorial convergente foi alcançada indicando convergência dos itens para cada um dos dois fatores e a validade discriminante se apresentou bem acima da correlação inversa entre os fatores, corroborando a bidimensionalidade do questionário. 
Quanto à confiabilidade (CR), o fator 2 (Dimensão 2) alcançou nível considerado satisfatório, mas o fator 1 (Dimensão 1) mostrou-se ligeiramente abaixo de níveis propostos por Brown ${ }^{8}$. No entanto, Maroco ${ }^{17}$ sinaliza que ao se avaliar um instrumento de mensuração "é importante que os valores estatísticos obtidos sejam sempre ponderados a partir de uma base teórica e metodológica, para que sua interpretação seja adequada" (p.224). Neste sentido, acredita-se que opções alternativas devam ser investigadas.

Faz-se necessário ressaltar que o estudo mostrou a necessidade de se ter trabalhado os dados para corroborar a bidimensionalidade do questionário. A natureza das dimensões (fatores) exige sub escalas separadas que sejam capazes de distinguir o construto "Adoção do óleo vegetal in natura de acréscimo na dieta infantil". Pode-se arguir que o processo de desenvolvimento do questionário não se completou tendo oito itens, mas é sustentável como uma abordagem do construto no contexto onde foi aplicado, sendo necessárias validações para outros contextos.

Considerando outras evidências favoráveis do Modelo D: AFC que foram obtidas (cargas fatoriais altas, variâncias de erros baixas, um único MI baixo), a média dos escores brutos foi considerada como forma de avaliar o comportamento dos responsáveis pela alimentação de crianças menores de dois anos, tarefa que não é tão simples. A utilização da média dos escores foi fundamentada na normalidade multivariada da distribuição dos escores dos itens.

O questionário "Adoção do óleo vegetal in natura de acréscimo na dieta infantil" foi elaborado para ser respondido pelos responsáveis pela alimentação da criança e inclui questões que avaliam tanto aspectos positivos "Benefícios do acréscimo de óleos vegetais in natura na dieta infantil" quanto os negativos "Possíveis problemas relacionados ao acréscimo de óleos vegetais in natura na dieta infantil" relativo a uma prática dietética recomendada pelo Ministério da Saúde, o que Ihe confere um caráter inovador devido à inexistência de alguma proposta alternativa. Em nosso conhecimento, este é o primeiro estudo que desenvolve um percurso metodológico para a elaboração e validação de um instrumento de mensuração em dietética destinado a avaliar o comportamento de responsáveis pela alimentação infantil em relação à adoção do óleo vegetal in natura de acréscimo na dieta infantil. Avaliou-se a dimensionalidade de um instrumento em um contexto de exploração e confirmação de análises que até então não haviam sido investigados em estudos de recomendação 
dietética para grupos de risco na infância sobre o uso do óleo vegetal in natura de acréscimo. Do ponto de vista da orientação nutricional, os escores sugeridos podem ser aplicados para ajudar a identificar como os responsáveis pela alimentação de crianças reagiriam em relação à referida prática. Sabe-se ainda necessária a divulgação ampla do mesmo, mas parece razoável reforçar sua divulgação como instrumento na prática de atendimento nas Unidades de Saúde e sua pontuação bruta como um "mapeador" de primeira abordagem de um continum subjacente. No entanto, a resposta sobre se uma estrutura fatorial será repetível em diferentes cenários de situações culturais requer novas evidências

\section{CONCLUSÃO}

As análises permitiram reduzir o número de itens de onze para oito e confirmaram a bidimensionalidade do questionário. O questionário reduzido alcançou tanto a validade fatorial convergente quanto a discriminante. A confiabilidade foi satisfatória para a Dimensão 2 - "Possíveis problemas relacionados ao acréscimo de óleos vegetais in natura na dieta infantil" e, com certa parcimônia, para a Dimensão 1- "Benefícios do acréscimo de óleos vegetais in natura na dieta infantil". No entanto, ainda que necessária sua validação em outros contextos, o questionário parece estar bem adequado para avaliar se os responsáveis pela alimentação da criança terão disposição para aderir ao uso do óleo vegetal in natura de acréscimo na dieta infantil.

\section{CONSIDERAÇÕES FINAIS}

A AFC via MEE é uma técnica que traz várias possibilidades para o desenvolvimento teórico e metodológico no campo de desenvolvimento de instrumentos de mensuração em saúde, no entanto possui limitações em seu uso. A MEE faz parte da família de técnicas multivariadas pertencentes ao modelo linear geral. Desta feita, o pesquisador deve lançar mão de alternativas que possam lidar com esse tipo de situação. A MEE é um caso cujos estimadores devem pressupor normalidade multivariada dos dados, que por consequência, levam ao tamanho de amostras menores se comparada àqueles dados que têm livre distribuição. Foi neste sentido que o estudo utilizou um teste de assimetria e curtose para testar a 
normalidade multivariada entre as distribuições dos escores utilizados. Essa perspectiva de modelagem teórica e de processo de análise de dados já está presente há certo tempo nas discussões da literatura de MEE, mas a aplicação mais frequente se tem visto mais recentemente. No entanto, não deixam de existir questões remanescentes que defendam a revisão do modelo e que se discutam como novos itens podem vir a cobrir característica latente em termos de intensidade e reprodutibilidade num espectro mais amplo de grupos sócio culturais.

Estudos sobre a associação entre comportamento alimentar e recomendações dietéticas advindas de Políticas de Saúde Públicas possibilitam que profissionais de saúde se beneficiem com o uso de escalas de mensuração validadas para conhecer a adoção de tais indicações. Neste sentido, os resultados deste estudo podem ser considerados indicadores da invariância psicométrica do questionário provisoriamente, mas que aguardam análises em outros contextos. Além desta vertente pragmática, a contribuição deste estudo prende-se que a AFC via MEE pode aprimorar modelos de mensuração, o que beneficia o desenvolvimento de modelos teóricos relevantes e eficientes para a descrição comportamental. Como toda técnica não muito difundida, a MEE merece atenção de pesquisadores para que problemas encontrados em decorrência do processo do desenvolvimento de instrumentos de mensuração possam ser evitados, esta talvez, seja a principal contribuição do estudo.

\section{REFERÊNCIAS}

1.Streiner DL, Norman GR. Health measurement scales: A practical guide to their development and use. 4th ed. Oxford, United Kingdom: Oxford University Press; 2008. 2. Brasil. Ministério da Saúde (MS). Dez passos para uma alimentação saudável: Guia Alimentar para crianças menores de 2 anos. $2^{a}$ edição. Brasília: MS; 2013.

3.Dias LPP, Martins ICVS, Cordeiro KS, Nunes JDC. Ácidos graxos essenciais ômega-3 e ômega-6 no leite materno e sua associação com o desenvolvimento infantil: revisão de literatura. Femina 2014;42 (5):225-228.

4.Jorge N, Lopes MRV. Determinação de compostos polares totais em óleos e gorduras de frituras. Hig Aliment 2005;19(134):46-50. 
5.Lanzillotti HS, Barros ME, Afonso FM, Barbosa RMS, Barreto FL. Estrutura fatorial do questionário de adoção do óleo vegetal in natura de acréscimo na dieta infantil. Cien Saude Colet 2019;24(2):465-474.

6.Doornik JA, Hansen $\mathrm{H}$. An omnibus test for univariate and multivariate normality. Oxford Bulletin of Economics and Statistics 2008;70(Supl. 1):927-939.

7.Kline RB. Principles and Practice of Structural Equation Modeling. 2th ed. New York, NY: The Guilford Press; 2005

8.Brown TA. Confirmatory factor analysis for applied research. New York: The Guilford Press; 2006.

9.Stata Corp LP, College Station [Computer program]. Version 12: Stata Structural Equation Modeling Reference: manual release 12. College Station: Stata Press Publication; 1985-2011.

10.Pilati R, Laros JA. Modelos de Equações Estruturais em Psicologia: Conceitos e Aplicações. Psic: Teor e Pesq 2007;23(2):205-216.

11.Browne MW, Cudeck R. Alternative ways of assessing model fit. In Testing structural equation models. London: Bollen KA, Long JS. Sage Publications Inc; 1993. 12.Sijtsma K. Reability beyond theory and into practice. Psychometrika 2009; 74(1):169-173

13.Tucker LR, Lewis $C$. A reliability coefficient for maximum likelihood factor analysis. Psychometrika 1973;38:1-10.

14.Marsh HW, Muthén B, Asparouhov A, Lüdtke O, Robitzsch A, Morin AJS, Trautwein U. Exploratory Structural Equation Modeling, Integrating CFA and EFA: Application to Students Evaluations of University Teaching. Struct Equ Modeling 2009; 16:439-476. 15. Hair JF, Black WC, Babin BJ, Anderson RE, Tatham RL, editors. Multivariate data analysis. 6th ed. Upper Saddle River: Pearson Prentice Hall; 2006.

16. Bonfim CB, Santos DN, Menezes IG, Reichenheim ME, Barreto ML. Um estudo sobre a validade de construto da Parent-Child Conflict Tactics Scale (CTSPC) em uma amostra populacional urbana do Nordeste brasileiro. Cad Saúde Pública 2011; 27(11):2215-2226

17.Maroco J, Oliveira RA, Vicente CS. Análise Fatorial do Inventário de Burnout de Maslach (mbihss) em Profissionais Portugueses. Psic Saúde \& Doenças 2013; 14(1):152-167. 
18.Raykov T: Analytic estimation of standard error and confidence interval for scale reliability. Multivariate Behav Res 2001;37:89-103.

19.Fornell C, Larcker DF. Evaluating structural equation models with unobservable variables and measurement error. J Mark Res 1981;18(1):39-50. DOI:10.2307/3151312

20.Lanzilotti RS, Barros ME, Caravellas R, Lanzillotti HS. Questionário sobre o uso do óleo vegetal comestível in natura na dieta infantil: analise fatorial exploratória. Rev Inova Saude 2017;6(2):1-19.

21.Ornellas LH. Técnica Dietética: seleção e preparo de alimentos. 8ª Ed. Rio de Janeiro: Atheneu; 2007.

22.Azevedo E. Liberem a dieta. Demetra 2015;10(3)717-727. DOI: 10.12957/demetra.2015.13993. 